Rev. Biol. Trop., 47(3): 617-622, 1999

www.ucr.ac.cr www.ots.ac.cr www.ots.duke.edu

\title{
Tamaño gonadal de machos de Chaetodipus arenarius (Rodentia: Heteromyidae) durante un ciclo reproductivo en Baja California Sur, México
}

\author{
Patricia Cortés-Calva y Sergio Ticul Alvarez-Castañeda \\ Centro de Investigaciones Biológicas del Noroeste, S. C., Mar Bermejo No. 195. Col. Playa Palo Santa Rita, La Paz, \\ B. C. S., C. P. 23090. México. Tel: 5211253633 , Fax: 5211255343
}

Recibido 15-VII-1998. Corregido 19-1-1999. Aceptado 2-II-1999.

\begin{abstract}
The description of reproductive activity in small nocturnal mammals is difficult. In this study we use the variation of testis characteristics over time. From February through December 1993 we set 1628 traps/night for the capture of specimens. We were studying the variation of testis size of 30 Chaerodipus arenarius sublucidus, and analyzing the morphometric characteristics and morphology of the sperm of this species compared with $C$. spinatus. We recorded the abundance of sperm per age. The size of the testis was greater from February through September. during which there was reproduction activity, and this corresponds to a larger amount of sperm. There were morphometric differences in the length size of sperm. The gonadal-somatic index indirectly indicated the reproductive activity.
\end{abstract}

Key words: Rodentia, Heteromyidae, reproduction, gonadal-somatic, sperm, Baja California Sur.

Parámetros biológicos como tamaño y peso de la gónada así como el número de tubos seminíferos, han sido utilizados para determinar la actividad reproductora en mamíferos pequeños (Baechman 1980, Faulkes et al. 1991, Moreira et al. 1997). La morfología y morfometría de los espermatozoides en mamíferos pequeños han sido criterios útiles para taxonomía y evolución de algunos géneros (Forman 1968). Acerca de la producción de esperma Moller (1989), menciona una relación directa con el tamaño del testículo, por lo que testículos grandes tienen una mayor producción de esperma, aportando una ventaja selectiva asociada a la inseminación múltiple (Harvey y Harcout 1984). En este estudio se evalúo la variación del tamaño testicular de machos de Chaetodipus arenarius sublucidus en un ciclo reproductivo, mediante índices gonado-somáticos, así como abundan- cia y morfometría de espermatozoides en diferentes clases de edad.

\section{MATERIALES Y MÉTODOS}

Los muestreos se efectuaron de febrero a diciembre de 1993 en tres localidades ubicadas al oeste de La Paz, Baja California Sur, México. "El Centenario" $\left(24^{\circ} 04\right.$ ' N y $110^{\circ}$ $\left.25^{\prime} \mathrm{W}\right)$, "El Comitán" (2405' N y $110^{\circ} 21^{\circ}$ ' W) y "Brisamar" (24․ $11^{\prime} \mathrm{N}$ y $\left.110^{\circ} 30^{\prime} \mathrm{W}\right)$. Con vegetación predominante de matorral sarcocaule (León de la Luz et al. 1996). El clima es cálido y árido seco (García 1981). Durante 1993 la temperatura media anual fue de $\bar{x}=24.0^{\circ} \mathrm{C}(7.5-40.5)$ y precipitación total anual de $176.7 \mathrm{~mm}$, la mayor precipitación ocurrió en septiembre $51 \mathrm{~mm}$ y noviembre con $27 \mathrm{~mm}$. El esfuerzo de captura fue de 1628 
trampas/noche. A los heterómidos machos capturados se les extrajo el aparato reproductor, cada gónada (incluyendo el epidídimo) fue pesada (g) y medida $(\mathrm{mm})$, posteriormente fijados en formol al 10\%. Se aplicaron índices de volumen testicular (Jones 1970; Estrada-Flores et al. 1990) e índices gonado-somáticos (Guillete y Casas-Andreu 1980).

Volumen testicular $V=4 / 3 \pi \mathrm{a}^{2} \mathrm{~b}$. Donde $\mathrm{a}=1 / 2$ del ancho del testículo y $\mathrm{b}=1 / 2$ del largo de la gónada.

IGS-P Indice gonado-somático de peso. Relación porcentual entre el peso de la gónada (incluyendo el epidídimo) y el peso del organismo.

Para la obtención del esperma, se maceró el epidídimo junto con un mililitro de solución fisiológica. Se calculo el número de espermatozoides en una alícuota de $\left(0.1 \mathrm{~mm}^{3}\right)$ capacidad de la cámara de Neubauer, considerando como referencia las cabezas de los espermatozoides dentro del área de la cuadrícula grande de la cámara.

Para la descripción de la morfología y morfometría de los espermatozoides de Chaetodipus arenarius sublucidus y $C$. spinatus peninsulae (especie simpátrica), se elaboraron laminillas de tinción con giemsa (Watson 1975), realizando la medición de 30 espermatozoides por especie, las medidas se registraron con base al criterio establecido por Forman (1968). Se aplicó una prueba estadística t student. Las clases de edad (adulta, subadulta y viejos) se determinan con base al desgaste del tercer molar superior (Schmidly 1972).

\section{RESULTADOS}

'En la recolecta anual se observó mayor proporción de machos (60.4\%) que a hembras (39.5\%), exceptuando agosto con sólo una hembra con presencia de embriones y abril donde no se capturaron ejemplares de la especie. Los valores de las medidas de las gónadas, obtenidos en ocho meses reflejan el cambio en el tamaño de febrero a septiembre, en este pe- riodo fue cuando los testículos tuvieron un tamaño mayor; mientras que el índice gonadosomático de peso expresa la disminución del peso corporal y muy posiblemente un aumento en peso de la gónada.

De marzo hasta julio que se mantiene sin cambios notorios y valores constantes, para los meses restantes la relación fue disminuyendo (Fig. 1).

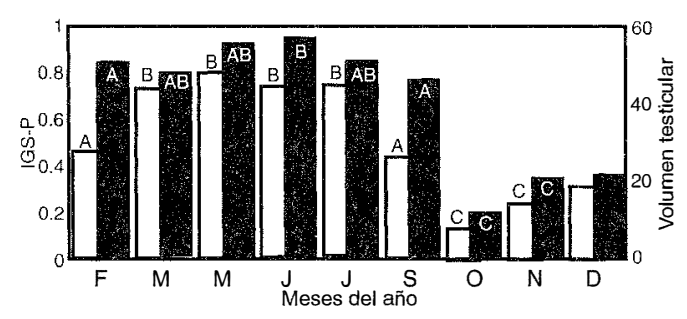

Fig. 1. Valores porcentuales del índice gonadosomático de peso (IGS-P, barras claras) y volumen testicular (barras achuradas); se denota el grado de significancia con letras A es significativamente diferente a B, y B significativamente diferente de $\mathrm{C}$. Value (percent) of gonadosomatic index weight (IGS-P, bright bar) and testicular volume (shady bar). The letters represent significance A is significantly different from B, and B is significantly different from C.

Morfológicamente la cabeza del espermatozoide es asimétrica (forma de gancho), sin procesos postacrosómicos, el cuello no se diferencia claramente, la región media ocupa casi el $40 \%$ de la longitud total, mientras que la región de la cola es más corta (Fig. 2). No se apreciaron malformaciones. Existieron diferencias en el tamaño entre los espermatozoides de $C$. arenarius y $C$. spinatus. La longitud total de $C$. spinatus es significativamente mayor (t student $\mathrm{P}<0.01$ ), mientras que la cabeza de C. arenarius es mas ancha (t student $\mathrm{P}<0.001$ ). La longitud media del cuerpo es diferente, apreciándose un tamaño mayor en C. spinatus. La longitud del flagelo y de la cabeza no mostró diferencias (Cuadro 1).

La valoración de la cantidad de esperma de $C$. arenarius se realizó de febrero a septiembre, observándose en mayo el valor mas bajo de producción espermática 650000 $\mathrm{cel} / \mathrm{ml}$. En junio y julio los valores sobrepasaron 


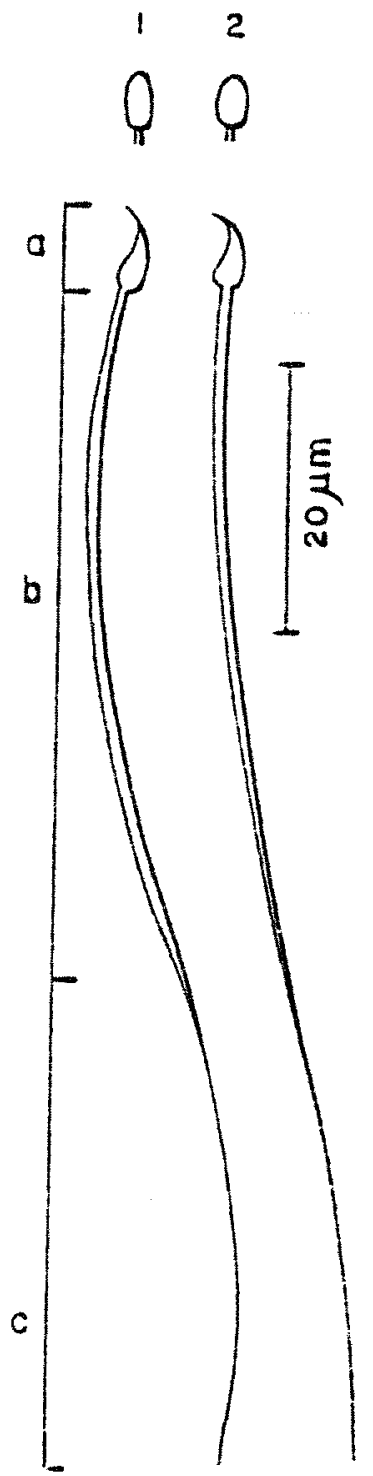

Fig. 2. Espermatozoides de 1.- Chaetodipus arenarius sublucidus, 2.- Chaetodipus spinatus peninsulae. Vista lateral, a) cabeza, b) región media, c) cola: vista dorsal de la cabeza. Spermatozoa of 1.- Chaetodipus arenarius sublucidus, 2.- Chaetodipus spi atus peninsulae. Lateral view, a) head, b) midpiece, c) tail; dorsal view of head.

los $3500000 \mathrm{cel} / \mathrm{ml}$. El número de espermatozoides en las tres clases de edad fue: subadultos $\overline{\mathrm{x}}=1275000(1800000-750000$ $\mathrm{cel} / \mathrm{ml})$, adultos 2842864 (6 $500000-812$ $500 \mathrm{cel} / \mathrm{ml})$ y viejos $3382143(5125000-$ $650000 \mathrm{cel} / \mathrm{ml}$ ).
Hubo una relación significativa entre el volumen testicular y el promedio mensual de espermatozoides, de febrero a septiembre $\left(\mathrm{r}^{2}=0.76 ; \mathrm{P}<0.005\right)$; no siendo así para: longitud del roedor y el volumen testicular $\left(\mathrm{r}^{2}=0.0048\right)$; longitud del roedor y longitud del testículo $\left(\mathrm{r}^{2}=0.0035\right)$; longitud del roedor y el peso de la gónada $\left(\mathrm{r}^{2}=0.0128\right)$; peso del roedor y el volumen de la gónada $\left(r^{2}=0.0334\right)$; peso del roedor y el peso de la gónada $\left(r^{2}=0.017\right)$.

\section{DISCUSIÓN}

Las variaciones en el tamaño de la gónada y producción de espermatozoides son características que infieren el patrón de reproducción de las especies. Según Harvey y Harcout (1984) y Moller (1989) los testículos más grandes tienen una mayor producción de esperma, aportando una ventaja selectiva asociada con la inseminación múltiple. Obteniéndose para esta especie que el mayor tamaño de los testículos fue de febrero a septiembre, lo que indicaría que es el periodo donde ocurre la cópula de $C$. arenarius, quedando referido como una relación directa entre el volumen testicular y el número de espermatozoides encontrados.

Los machos de Chaetodipus arenarius tienen un comportamiento similar a lo registrado por Brown et al. (1979) y Munger et al. (1983), quienes señalan que los factores ambientales influyen en el desarrollo y frecuencia copulatoria de los heterómidos. Para C. arenarius el mayor tamaño de las gónadas coincide con la época seca del año en la región, existiendo abundancia de semillas de diversas plantas anuales y perennes (León de la Luz et al. 1996), de lo que se especula que este fenómeno es una estrategia para que los nacimientos ocurran en la época de mayor abundancia de las semillas (Price 1978, Munger et al. 1983).

Los espermatozoides de Chaetodipus son mas pequeños, en comparación al tamaño promedio señalado para los de Tilomys y Ototylomys (Helm y James 1973), Peromyscus 


\section{CUADRO 1}

Medidas morfométricas de los espermatozoides de Chaetodipus arenarius sublucidus $(n=30)$ y Chaetodipus spinatus peninsulae $(n=30)$ Morphmetrics meastrements spermatozoa of Chaetodipus arenarius sublucidus $(n=30)$ and Chaetodipus spinatus peninsulae $(n=30)$

$\begin{array}{llcccc}\text { Medidas } & \text { Especie } & \text { Media } & \text { D.E. } & \text { Máxima } & \text { Mínima } \\ \text { Long. total } & \text { C. spinatus } & 122.5 & 5.24 & 1.24 .48 & 120.58 \\ & \text { C. } \text { arenarius } & 118.5 & 9.7 & 121.11 & 115.49 \\ \text { Long. cabeza } & \text { C. spinatus } & 5.76 & 0.72 & 7.23 & 4.29 \\ & \text { C. } \text { arenarius } & 5.75 & 0.42 & 5.77 & 5.73 \\ \text { Long. media } & \text { C. spinatus } & 56.85 & 9.88 & 60.53 & 53.17 \\ & \text { C. } \text { arenarius } & 48.72 & 7.37 & 53.38 & 44.06 \\ \text { Long. cola } & \text { C. spinatus } & 62.54 & 10.25 & 63.72 & 61.36 \\ & \text { C. } \text { arenarius } & 63.75 & 9.7 & 66.57 & 60.92 \\ \text { Ancho cabeza } & \text { C. spinatus } & 2.92 & 0.34 & 2.93 & 2.79 \\ & \text { C. } \text { arenarius } & 3.3 & 0.31 & 3.31 & 3.29\end{array}$

D.E. = desviación estándar de la muestra

(Linzey y Layne 1974) y Mus (Houillon 1978). Los meses de mayor abundancia de espermatozoides (mayo, junio y julio) coincidieron con el aumento del tamaño de los testículos, la que coincide con los datos de preñez registrados de mayo a agosto para hembras de la misma especie en la región (Cortés-Calva y Alvarez-Castañeda 1997), aclarando que la frecuencia copulatoria no es un parámetro que se haya controlado en este estudio. Ahora bien al establecer la relación de la producción esperma en las distintas clases de edad, se observó que sí existe variación, ya que aumenta con la edad, existiendo una relación directa conforme a las tres clases de edad, las que consideramos aptas para la reproducción, coincidiendo con lo publicado por Squires et al. (1979).

Podemos deducir que la variación en el volumen testicular está relacionada con la producción de esperma, siendo una consecuencia de la posible frecuencia copulatoria del roedor y de las tácticas reproductoras que presentan en la población. En los machos adultos, el yolumen de la gónada se mantiene constante, existiendo aumento de tamaño en la época seca del año (febrero a septiembre). El úndice somático de peso mostró que el peso corporal de los individuos varía a lo largo del tiempo, en términos energéticos, la energía destinada para la reproducción no se ve alterada, ya que el peso de la gónada es constante e independiente al peso del organis- mo. Mientras que la abundancia de los espermatozoides esta altamente relacionada con el volumen testicular, además de presentar una relación positiva con la edad, debido a que la mayor cantidad se obtuvo en organismos fisiológicamente maduros y aptos para la reproducción.

\section{AGRADECIMIENTOS}

Agradecemos el apoyo al proyecto 012PÑ-1297 CONACyT, así como a Ellis Glazier por la revisión del idioma inglés.

\section{RESUMEN}

El patrón de reproducción de roedores nocturnos es difícil de describirse en condiciones naturales, para inferirse se recurrió a las características de la gónada a través del tiempo. De Febrero a Septiembre de 1993, colocándose 1628 trampas/noche para la captura de roedores. Se estudió la variación del tamaño testicular de machos de Chicetodipus arenarius sublucidus, además se analizó la morfometría y morfología de espermatozoides de 30 C. arenarius comparándose con la de $30 \mathrm{C}$. spinatus. $\mathrm{Al}$ mismo tiempo se registró la abundancia por clases de edad. El tamaño de los testículos fue mayor de febrero a septiembre, época en la que ocurre la actividad reproductora, y que se relacionó con uná mayor cantidad de espermatozoides. Existió diferencia morfométrica de los espermatozoides de lạs dos especies. Los índices gonadosomáticos advierten indirectamente la actividad reproductora. 


\section{REFERENCIAS}

Baeacham, T. D. 1980. Breeding characteristics of Townsend's vole (Microtus towsendii) during population fluctuations. Can. J. Zool. 58:623-625.

Brown, J.H., D.W. Davidson y O. J. Reichman. 1979. An experimental study of competition between seed-eating desert rodents and ants. Amer. Zool. 19:1129-1143.

Cortés-Calva, P. y S. T· Alvarez-Castañeda.1997. Estimación y número de camada de Chaetodipus arenarius (Rodentia:Heteromyidae) en Baja California Sur, México. Rev. Biol. Trop. 44:301-304.

Estrada-Flores, E., M. Villagran-Santa Cruz., F. R. Mendez-De La Cruz. y G. Casas-Andreu. 1990. Gonadal changes througout the reproductive cycle of the viviparous lizard Sceloporus mucronatus (Sauria: Iguanidae). Herpetology 46:43-50.

Faulkes, C.G., D.H. Abbott y J. U. M. Jarvis. 1991. Social suppression of reproduction in male naked mole-rats, Heterocephalus glaber. J. Rep. Fert. 91:593-604.

Forman, G. L. 1968. Comparative grass morphology of spermatozoa of two families of North American bats. Univ. Kansas Sci. Bull. 47:901-928.

García, E. 1981. Modificaciones al sistema de clasificación de Köppen. Inst. Geogr. Univ. Nal. Autón. Mex. México. 1-217.

Guillette, L. J., Jr. y G. Casas- Andreu. 1980. Fall reproductive activity in the high altitude Mexican lizard Sceloporus grammicus microlepidotus. J. Herpetol. 14:143.

Harvey, P. H. y A. H. Harcout. 1984. Sperm competition, testes size, and breeding system in primates. P. 589600. In Smith, R. L. (ed.). Sperm Competition and the Evolution of Animal Mating Systems. Academic Press, Nueva York.

Helm, J. D. y J. R. Bowers. 1973. Spermatozoa of Tylomys and Ototylomys. J. Mamm. 54:769-772.
Houillon, C. 1978. Sexualidad. Omega. Barcelona, España, p.13-136.

Jones, R. E. 1970. Effect of season and gonadotropin on testicular interstitial cell of California quail. Auk 87:729-737.

León de la Luz, J. L., R. Coria y M. E. Cruz. 1996. Fenología floral de una comunidad árido-tropical de Baja California Sur, México. Acta Bot. Mex. 35:45-64.

Linzey, A. V. y J. L. Layne. 1974. Comparative morphology of spermatozoa of the rodent genus $\mathrm{Pe}$ romyscus (Muridae). Amer. Mus. Novitates 2355:1-20.

Moller, A. P. 1989. Ejaculate quality, testes size and sperm production in mammals. Funct. Ecol. 3:91-96.

Moreira, J. R., J. R. Clarke y D.W. Macdonald. 1997. The Testes of capybaras (Hydrochoerus hydrochaeris). J. Mamm. 78:1096-1100.

Munger, J. C., M. A. Bowers y W.T. Jones. 1983. Desert populations: factors affecting abundance, distribution and genetic structure. p. 91-116. In Reichman, O.J. y J.N. Brown (eds) Biology of desert rodents. Great Bas. Nat. Mem.7:1-134.

Price, M. V. 1978. The role of microhabitat in structuring desert rodent communities. Ecology 59:910921.

Schmidly, D. J. 1972. Geographic variation in the white ankle mouse, Peromyscus pectoralis. Southwest. Nat. 17:113-138.

Squires, E. L., B. W. Pickett y R. P. Amann. 1979. Effect of successive ejaculation on stallion seminal characteristics. J. Rep. Fertil (suppl). 27:7-12.

Watson, P. F.1975. Use of Giemsa stain to detect changes in acrosomes of frozen ram spermatozoa. Vet. Rec. 97:12-15. 